\title{
Advanced EVA Roadmaps and Requirements
}

\author{
Richard K. Fullerton \\ NASA Johnson Space Center
}

\begin{abstract}
A wide range of solutions can be theorized for the architectures, technologies and operations concepts of advanced EVA systems for future space and planetary applications. This paper reports on the status of the latest advanced EVA roadmaps and ongoing work to capture and refine an initial set of candidate

requirements. A brief summary of related research and development is also presented. The end goal is a cost effective, safe and resource efficient integrated system that enables work in a diversity of environments at multiple destinations. A balance of cooperative efforts between humans and automated/robotic devices is envisioned to maximize productivity and safety in remote locations. The challenges ahead to be addressed will reflect past lessons learned and visionary new solutions. This information is intended to provide credible and useful guidance for those involved in the eventual implementation, validation and utilization of an advanced EVA system.
\end{abstract}

\section{INTRODUCTION - WHY HUMANS?}

The debate over the selection of human versus robotic means to accomplish space exploration is long standing and will surely continue. Each approach has pros and cons depending upon the intended application and the state of technology readiness. It is readily acknowledged that robotic missions are entirely appropriate for distant and hazardous new environments. At some point however, a combination of human and robotic resources provides leverage to enable more productive and timely efforts. This joint approach has numerous benefits that can be applied to a diversity of future exploration destinations and commercial ventures. Human intervention at a given site provides specific positive gains.

- Productivity - Use of creative cognitive abilities for rapid on scene decisions which overcome radio communication time delays and bandwidth limitations

- Reliability - Additional capability for response to unforeseen situations and unique non-repetitive activities

- Cost/Mass - Less need to expend resources upon complex, redundant and fully automated designs

- Terrestrial Benefits - Human space activities engage public interest and advance new opportunities
These human capabilities are further enhanced when appropriate tools are advantageously applied. Environmental protection, transportation vehicles, sensors, computerized information processing and mechanical handling aids typify classes of such aids. Interactive robotics also provide complementary strength, intelligence and extended duration external access. Direct teaming of the human brain and these aids has historically proven to be an effective means to enable difficult or otherwise impossible ventures.

\section{CURRENT EVA LIMITATIONS}

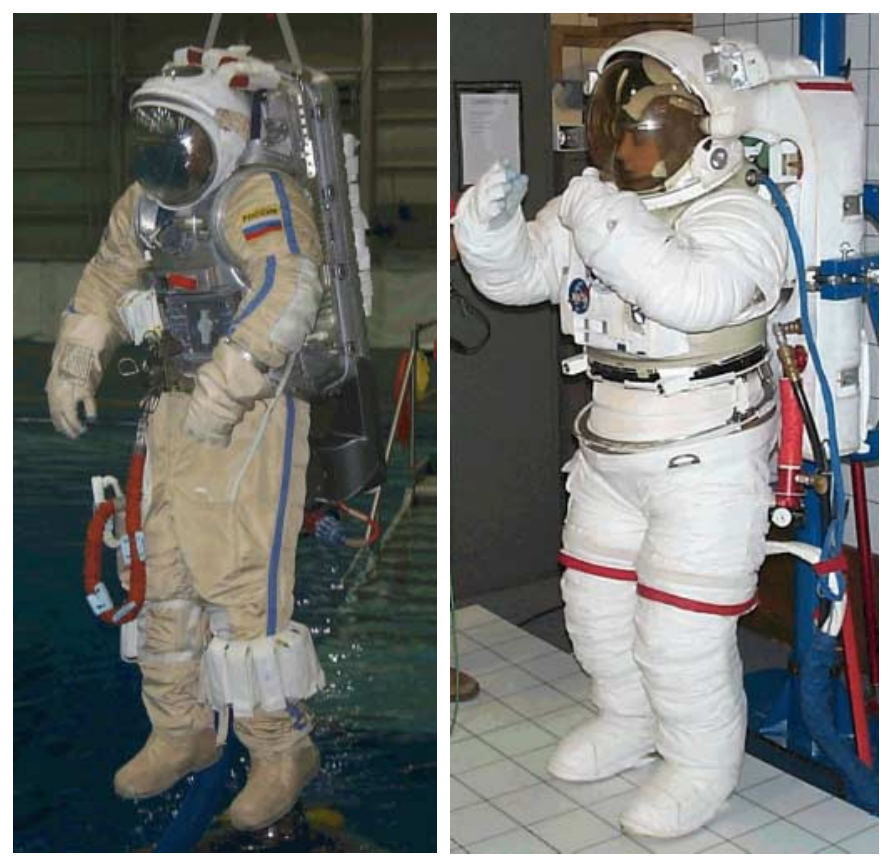

FIGURE 1. Russian Orlan-M and U.S. EMU

One tool that enables humans to work productively and effectively in space is the extravehicular activity (EVA) suit. Its origins are rooted in high altitude flights where protection from extreme cold and low pressure was paramount. Work compatible designs have culminated in the U.S. extravehicular mobility unit (EMU) and in the Russian Orlan M suits. While these suits are proven and robust to meet near term applications such as the International Space Station, they have serious limitations, which need to be addressed. The current NASA EVA suit design baseline is over 24 years old 
(1977) and has evolved from Apollo, Skylab and Shuttle program applications. It is only compatible with low earth orbit and microgravity activities. It requires regular ground based maintenance, re-supply and monitoring. Obsolescence of materials and components is an ongoing challenge. It relies upon a rigid architectural platform that is not well suited for advanced technology upgrades.

A synopsis of key issues with both U.S. and Russian EVA systems can be broken down into environmental, productivity and logistics induced factors :

Environmental Issues

1. The mass, mobility and visibility of the current suits are not compatible with partial gravity planetary environments. Suited body control in zero gravity is also hampered by these factors. The current U.S. suit is twice as heavy as the Apollo suit and is not designed for kneeling, prolonged walking or inertia free handling. Arm/hand work envelope and foot visibility are severely degraded by chest-mounted controls. Physical comfort is not sustainable for high frequency work in partial gravity.

2. Suit protection from dust intrusion is inadequate. Even the Apollo suits would have been unable to support more than 3 days of lunar work due to highly abrasive minerals preventing rotation of mobility bearings.

3. Available thermal insulation materials either only work in vacuum conditions or are thick and impede suit mobility and glove dexterity. Even with active heating, touch temperatures are limited to short durations and narrow ranges $\left(-140\right.$ to $+240^{\circ} \mathrm{F}$ or -96 to $\left.116^{\circ} \mathrm{C}\right)$.

4. Radiation environment definition, monitoring and protection are inadequate beyond earth's ionosphere.

5. The effects of planetary unique gases (such as argon) on EVA physiology are undefined.

6. Sensitive environments and science devices are contaminated from suit by-products (water, particulates, atmosphere leakage).

Productivity Issues

1. EVA information processing is limited to suit/medical telemetry and is based on old technology that is not inflight reprogrammable. Radio communication is the sole means of information exchange for science interaction, worksite unique data and navigation/tracking status. Imagery is only captured by standard photography and video. Reference information is paper based because no environment compatible display yet exists. Hands free interaction is needed to avoid fatiguing manual efforts and obstructed work volumes.

2. Medical monitoring and treatment of EVA crew is minimal. Cannot yet quantitatively track fatigue or decompression sickness symptoms. Non-intrusive, $100 \%$ O2 compatible and wireless devices are lacking. There is no effective insuit treatment capability for injury or illness.

3. Robotic EVA aids in use are primarily large arms with limited mobility and dexterous capability. Human capable wheeled rovers are not in development. Highly mobile and dexterous robotics get limited attention.
None are yet fully developed for autonomous inspections, cargo handling, worksite setup, crew tracking or self charging/storage/maintenance. Most are too reliant upon unique visual and handling aids.

4. Tools are limited to manual force/torque reaction \& zero$\mathrm{G}$ transport/restraint. Limited environmental \& mechanical analysis devices. No drills. Few true repair options. Delicate materials not easily handled.

Logistics Issues

1. EVA overhead penalties are high in terms of mass, volume and time. Historically, less than $20 \%$ of crew time related to EVA is spent on productive external work. $2600 \mathrm{lbs}$ and $90 \mathrm{ft} 3\left(1182 \mathrm{~kg}\right.$ and $\left.2.6 \mathrm{~m}^{3}\right)$ were manifested for suits, tools, carriers and consumables on STS-103 for Hubble Space Telescope servicing (1470 $\mathrm{lbs}$ and $60 \mathrm{ft} 3$ or $668 \mathrm{~kg}$ and $1.7 \mathrm{~m}^{3}$ for 4 suits). The 300 $\mathrm{lb}$ mass and $13 \mathrm{ft} 3\left(136 \mathrm{~kg}\right.$ and $\left.0.4 \mathrm{~m}^{3}\right)$ stowage volume of the current U.S. suit is not compatible with the restricted delivery capacity of remote exploration.

2. Suit consumables are wastefully expended and require frequent replenishment or considerable time/power to recharge. Heavy cooling water is vented. CO2 scrubbing canisters require wholesale replacement or time/power consuming bakeout between sorties. No insitu resource utilization is possible.

3. No real suit maintenance capability exists beyond limited resizing and consumables replacement. Spares change out is only done via large integrated assemblies. Many intricate parts are not crew serviceable.

4. Airlock designs have remained static. Depress/repress gas is still vented or pumped with large power penalties. Existing designs are not compatible with dust/biologic isolation or hyperbaric treatment.

5. Separate self rescue and emergency life support limits return range and adds to suit mass/volume

\section{IMPLEMENTATION GOALS AND REQUIREMENTS}

To enable the human and robotic aspects of efficient and effective space ventures, a visionary yet practical approach is planned. By documenting and maintaining a collection of the best known requirements from a broad set of sources, technology research and development will proceed with real targets in sight. Because past programs have suffered from late and incomplete collections of requirements, the hope is that a detailed and early capturing of this information will lead to success in future EVA implementation. Rather than wait for a specific destination to be named, a wide range of relevant and accessible environments will be targeted. The resulting products will be compatible with multiple destinations and provide an open architecture to enable a diversity of opportunities. Unlike the limited flexibility of the current technologies, a well thought out integrated system will be a readily adaptable and cost effective enhancement to human capabilities. As shown in the roadmap of Appendix A, an initial design can be fully implemented within 10 years and can improve as time and resources are further invested. Investing in a more efficient system will also save resources in the mid to long term. 
A draft document of the necessary requirements has been compiled from the best of numerous existing sources (individual experts, reports and past programs). To avoid painful and costly iterations, the mistakes and successes of the past will be heeded in future designs. This information will provide planners, designers and fabricators with a standard reference of the desired end products and uses. All significant EVA operations and hardware elements are considered. These include operations guidelines and hardware systems such as suits, airlocks, robotics, tools and ground infrastructure. Guiding priorities and principals include safety, simplicity, reliability, low mass, low cost, resource frugality, comfort, time efficiency and commonality. To maintain compatibility, vehicle and EVA elements are to be thought of as interdependent systems. Key to all designs is the ability to serve multiple uses without jeopardizing specific tasks.

The current edition of this requirements document is being maintained on a website at the Johnson Space Center at http://www.jsc.nasa.gov/xa/advanced.html. An outline of the draft document follows :

\subsection{Introduction}

2.0 Task Definitions and Operations Scenarios

3.0 Overall Resource Allocations/Needs

4.0 Strategic Groundrules, Constraints and Assumptions

5.0 Environments

6.0 Vehicle and Science Interfaces

7.0 Robotic Interfaces

8.0 Airlock

9.0 Suit/Umbilicals

10.0 Tools

11.0 Information Technology

12.0 Human Factors

13.0 Medical Constraints

14.0 Safety/Hazard Controls

15.0 Standards

16.0 Training/Development/Processing Facilities

17.0 Hardware Verification

Top level performance goals of the suit and its interfaces will be extremely challenging and will open up new horizons when achieved (refer to Appendix B). A 100 sortie usable life would grow from the current 25 sortie capability. The overall suit mass would shrink from $300 \mathrm{lbs}(136 \mathrm{~kg})$ to less than $80 \mathrm{lbs}(36 \mathrm{~kg})$. Though $\mathrm{O} 2$ recharge may need to be externally supplied, consumables self-sufficiency and regeneration is targeted to approach $100 \%$. Crew overhead time would be reduced from $80 \%$ of total EVA time to less than $50 \%$ and include no need for aid from shirt-sleeved crew. Ground support costs would decrease to $1 / 3$ of current levels. The overall cost of a production unit suit would be cut in half. Overall reliability, safety and comfort would be improved.

The ability to engineer these systems for work in extreme environments starts by understanding the basic characteristics of those environments. The adequacy of advanced EVA designs will be dependent upon feedback from robotic precursor investigations. Predicting natural radiation levels is the major open question for deep space locations. For asteroid and planetary sites, much remains to be learned. Knowledge needed includes surface,

subsurface and atmospheric parameters. Daily, seasonal and location/elevation variations in pressure, temperature, radiation, illumination, atmospheric composition and wind must be studied. The size, shape, composition, distribution, corrosion, abrasion and electrical charge properties of local dust are critical to reliable designs. Soil and rock

topography, mechanical strength, chemical composition/reactivity, thermal characteristics, electromagnetic properties and size/shape/distribution are of great interest. By better understanding these environments, the foundation of the implementation requirements will be strong and yield more capable products.

For each destination, mission scenarios and architectures continue to be devised. Recent studies have addressed options for Lunar, Martian, asteroid, Lagrangian and Earth orbital sites. For the planetary locations, a set of EVA traverse rules and rationale have been captured in an updated Mars Exploration Operations Concept book published by the Johnson Space Center (JSC). It relies upon lessons learned from past NASA missions and it balances expected new capabilities with the need for safe and successful remote excursions. The guidelines in this document are aimed for long duration missions where time is less of a constraint than sustained crew health and productivity. Included are rules for work priorities, surface acclimatization time/distance, minimum visibility with obscuring dust, night time navigation, radiation safe havens, fault tolerance and constraints for sortie frequency, duration and distance.

Demonstrations and tests are either ongoing or are planned to iteratively validate and refine these requirements. Terrestrial field tests, human factors assessments and technology research are conducted as resources permit (Appendix D). A build, test and iterate philosophy is being applied to downselect from the many implementation options. Expanded investigations into low and mid technology readiness levels will minimize the uncertainties of future implementation investments. Discrete high quality demonstrations on the ground and on-orbit are envisioned to validate topics sensitive to complex environments prior to such investments (e.g. gloves, robotics, information displays, materials, suit components/assemblies). In 5 to 6 years, a test validated multi-destination system could be ready for the implementation decision makers. Placing this system into active near earth service will further aid its refinement and reliability for remote uses.

The controlling authority for NASA's advanced EVA requirements is the JSC EVA Project Office Configuration Control Board (CCB). This forum is inclusive in nature and allows for a focused consensus of EVA requirements. It allows for representatives throughout JSC, NASA, industry, academia as well as international organizations.

Requirements revisions are foreseen to be necessary as new lessons are learned and as technology advances. The details of subsystem and component hardware requirements and verification will be delegated to lower documents managed by another level of control board. 


\section{ADVANCED EVA SYSTEM OVERVIEW}

The heart of a human EVA system continues to be an anthropometric, highly mobile and dexterous garment and life support system. For maximum access to difficult terrain or the confined work spaces of a vehicle, it is difficult to enclose the human form in a more efficient package. When supplemented with enhancing work aids, a truly capable tool for many applications can exist. The elements of an integrated external work system are diverse as can be seen in the system depiction of Appendix C.

There are currently at least 4 garment candidates available for near to mid term development. Two basic types of garments are depicted. The 3 atmospherically pressurized suits are differentiated by the amount of hard versus soft elements used to provide mobility/dexterity. The $4^{\text {th }}$ suit is basically a skin tight enclosure which uses mechanical pressure for protection from vacuum conditions. Each suit type is rooted in a long history of past research and development and is ready for further attention.

Crucial to any new suit garment are lightweight and durable materials. Besides compensating for low external pressures, these materials must also be dust, contamination and puncture resistant. Light weight, low bulk thermal insulation for vacuum and non-vacuum environments are mandatory. Materials capable of evaporative cooling and radiation protection are of interest to minimize the need for complex alternatives. In parallel with suit design, the impacts of low habitat pressures and new breathing gases must be studied to mitigate decompression sickness issues.
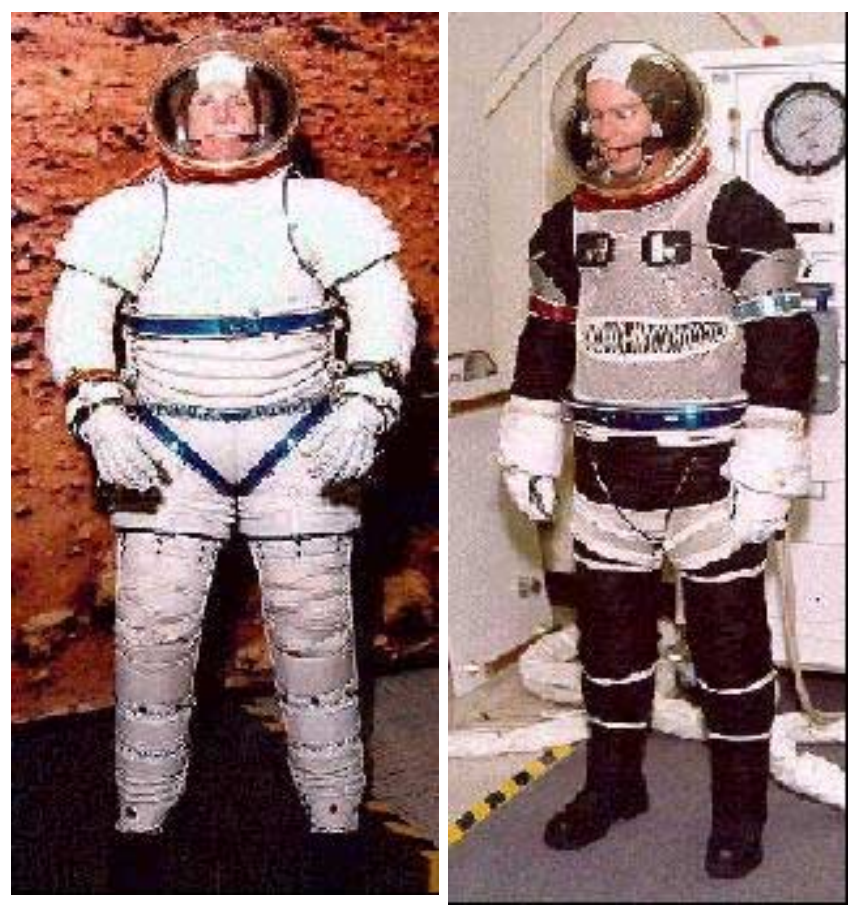

FIGURE 3A. Advanced EVA Garment Options (I and D)
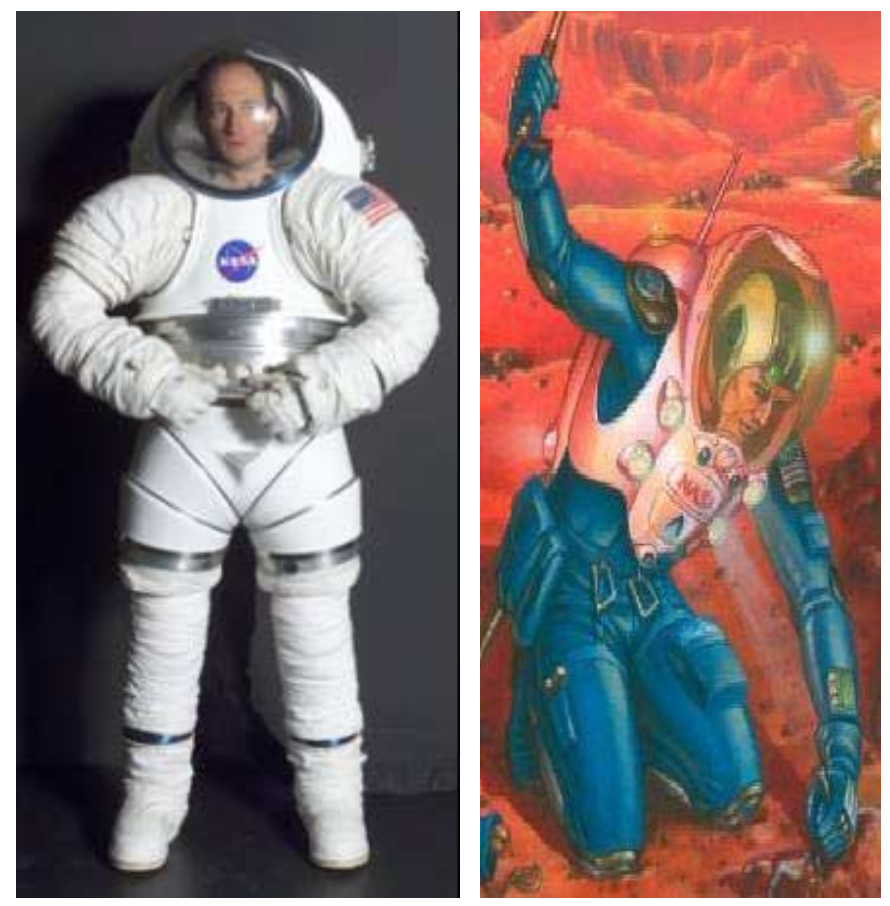

FIGURE 3B. Advanced EVA Garment Options (H and MCP)

To be truly mobile, self-contained life support systems must accompany the suit garment. While umbilicals have a role in selected localized applications, portable systems are still necessary. Because this equipment is by far the "long pole in the tent", it requires the most time and effort to develop. The priorities to be addressed include $\mathrm{CO} 2$ scrubbing, $\mathrm{O} 2$ storage/supply, active thermal control and integrated mass/volume reduction. Most of the advanced concepts feature passively regenerable $\mathrm{CO} 2$ removal, cryogenic liquid $\mathrm{O} 2$ and radiator cooling. Fuel cell based power can enable a multitude of compact wireless sensors/actuators, intelligent automated information systems and navigation / communication aids for internal and external interfaces. The arrangement of this system will require novel packaging for low weight, low volume and ease of maintenance.

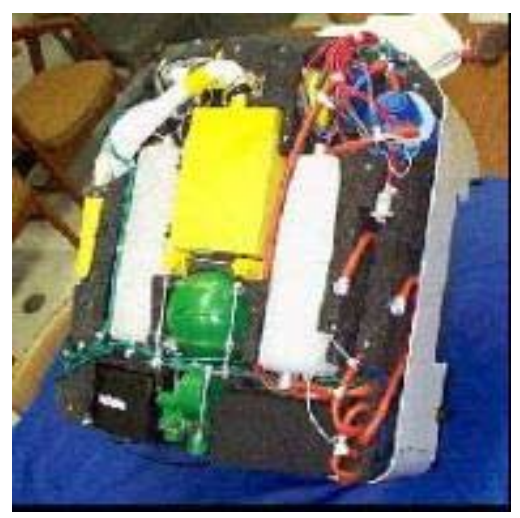

FIGURE 4. Conceptual Life Support Backpack 
A suite of support aids must also be developed to make the human in the suit safe, productive and effective. This includes airlock concept mockups for mobility testing of normal and incapacitated crew persons. Robotic and human interaction testing is needed to quantify relative merits and work assignments. To minimize mass and volume burdens upon mission architectures, airlock concepts of interest include inflatables and suit ports. Tools are needed for quantified comparative analysis of suit mobility, fit, sizing and design. Hands free heads up displays/controls projected onto the helmet's visor and married to environmental sensors/imagers are desired. Devices, which actively and artificially protect against harmful natural radiation need to be pursued. Mechanical aids that enhance the basic strength, skills and equipment handling capabilities of the suited human should be devised and integrated to enable otherwise impractical capabilities.

Because available resources are limited, the following topics are listed in their approximate order of technical priority.

1. Integrated Concept Definition and Requirements (suit, airlock, robotics)

2. $\mathrm{CO} 2$ system

3. Mass/Volume reduction and system definition (SSA and LSS)

4. O2 system

5. Environmental Protection (thermal, puncture, radiation, dust)

6. Thermal Control System

7. Test Personnel and Facilities

8. Analysis Tools

9. Power supply system

10. Instrumentation and info technology

\section{COMMERCIAL APPLICATIONS AND RELATED RESEARCH}

Multiple commercial applications are viable from EVA technology. From past experience, human work in space is a proven valuable tool for satellite servicing and the assembly and maintenance of large structures. Human and robotic teamwork can also facilitate space based manufacturing, power generation, tether based launch services and tourism. Terrestrial users benefit from commercialized EVA technologies as well (firefighter garments and breathing sources, underwater dive industry/tourism, pollution controls, submarine life support, medical sensors and protective garments). When space derived technology is commercially massproduced, it can be reapplied at reduced costs. As a microcosm of large scale human projects, overall costs could be reduced by up scaled utilization of EVA systems. Maximum leverage can and will be applied through commercial non-EVA projects (info technology, robotics, nano technology, power sources, medical devices). Such shared commonality adds to total reliability, maintainability and redundancy for all users.

\section{CONCLUSIONS}

EVA is a cross cutting infrastructure which is fundamental to enabling current and future exploration and commercial endeavors. While current technology serves the needs of low earth orbit, it is not suited to efficient and cost effective applications (nearby or distant). If sustained support is received, within 10 years a truly advanced and destination independent set of flight and training quality hardware can be ready to support existing and future programs. In half that time, a prototype system could be demonstrated for implementation decisions. With such investment, there is great potential to eliminate the high costs of maintaining current hardware thru less expensive new hardware and close inspection of current inefficiencies. Savings can be reinvested to ultimately free up ground/crew time, lower production/utilization costs and minimize resupply efforts while enhancing human productivity. Without ongoing investment, future generations will be challenged to devise human and robotic cooperative programs from the current low level efforts, which are neither efficiently productive nor sustainable.

\section{REFERENCES}

1. Advanced Technology For Human Support In Space, National Research Council, 1997

2. Good Use of Flight Crew Time-Guiding Principle for EVA System Design, 941556, 24 ${ }^{\text {th }}$ ICES, June 1994

3. ISS Phase 1 EVA Experience, 00ICES-212, $31^{\text {st }}$ ICES, July 2000

4. Kozloski, Lillian D., U.S. Space Gear, Smithsonian Institution Press, 1994

5. Space Biology and Medicine, volume II, chapter 14, Individual Systems for Crewmember Life Support and Extravehicular Activity, AIAA, 1994

\section{CONTACT}

Author richard.k.fullerton1@jsc.nasa.gov

JSC EVA Project Office http://www.jsc.nasa.gov/xa/advanced.html

JSC Crew \& Thermal Systems Division http://ctsd.jsc.nasa.gov/ESS/advanced.html

\section{DEFINITIONS, ACRONYMS, ABBREVIATIONS}

$\begin{array}{ll}\text { EMU } & \text { extravehicular mobility unit } \\ \text { EVA } & \text { extravehicular activity } \\ \text { ISS } & \text { international space station } \\ \text { IVA } & \text { intravehicular activity } \\ \text { MCP } & \text { mechanical counter pressure } \\ \text { LSS } & \text { life support system } \\ \text { N/A } & \text { not applicable } \\ \text { SSA } & \text { space suit system }\end{array}$




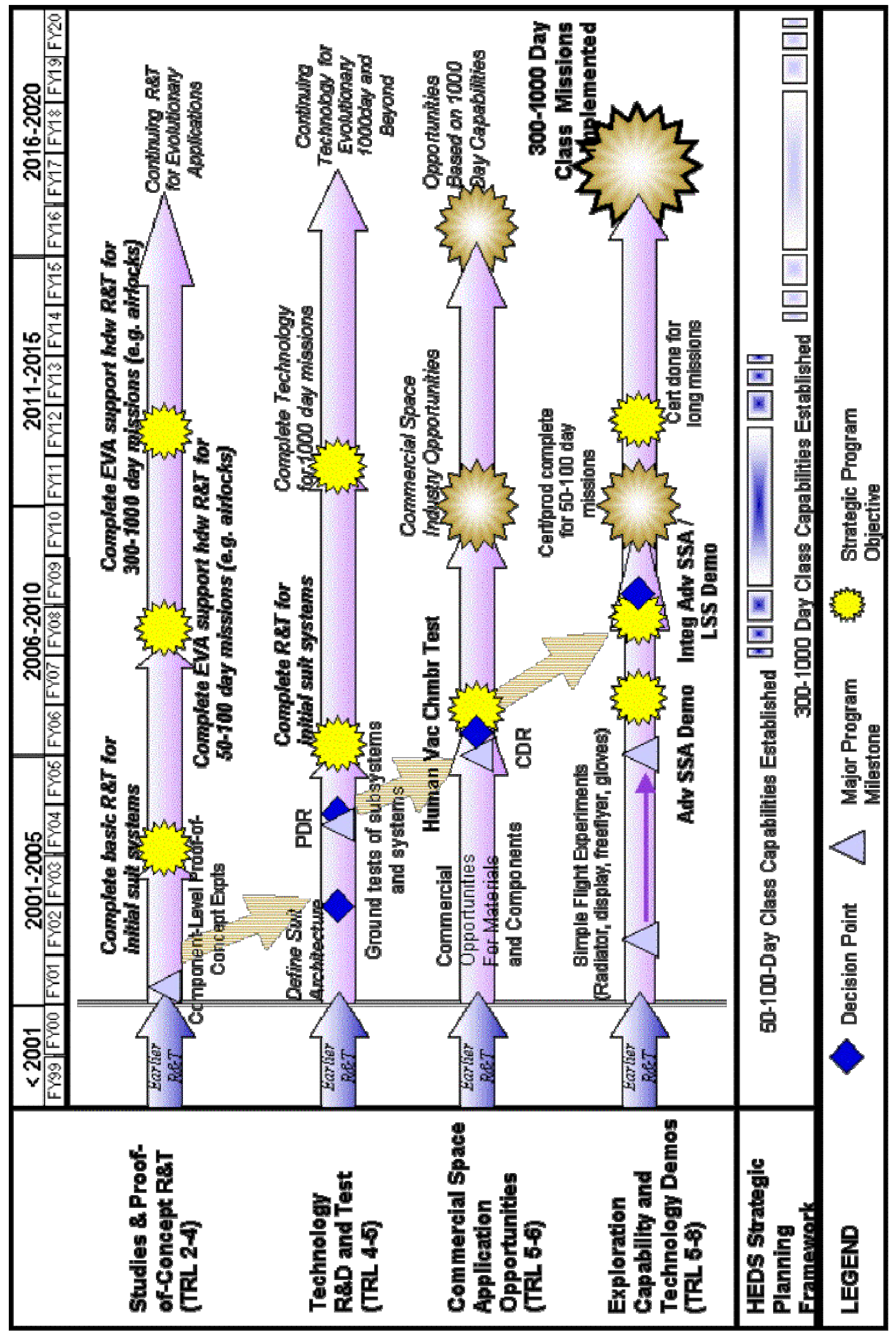


APPENDIX B - OVERVIEW OF ADVANCED EVA STRATEGIC TARGETS

Total Suit Weight $<80 \mathrm{lbs}$ ( $27 \%$ of current $300 \mathrm{lb}$ suit)

No fatigue or discomfort after single sortie

Backpack duration of 4-36 hours (incl recharge)

MCP, Soft or Hard/Soft Hybrid Garments

Reliability $>0.999 \mathrm{X}$

Suit lifetime $=100$ sorties

DCS, MMOD, radiation risk $<X \%$

Fail cafo roliahility > TRח

Consumables self sufficiency $>95 \%$

No suit consumables but $\mathrm{O} 2$

Airlock pwr $<X$ watts

Airlock aas loss $<X \%$

Crew Overhead Time $<50 \%$ (80\% today)

No tools for std interfaces

Zero IVA crew support

Pre and post EVA crew time $<1$ hour each

$\underline{\mathbf{5 0} \% \text { cost reduction per suit (post cert) }}$

Ground turnaround \& ops costs $<1 / 3$

Ground turnaround < TBD manhours per suit Ground Servicing Personnel < TBD EP

\section{APPENDIX C - ELEMENTS OF EXTERNAL WORK SYSTEM}

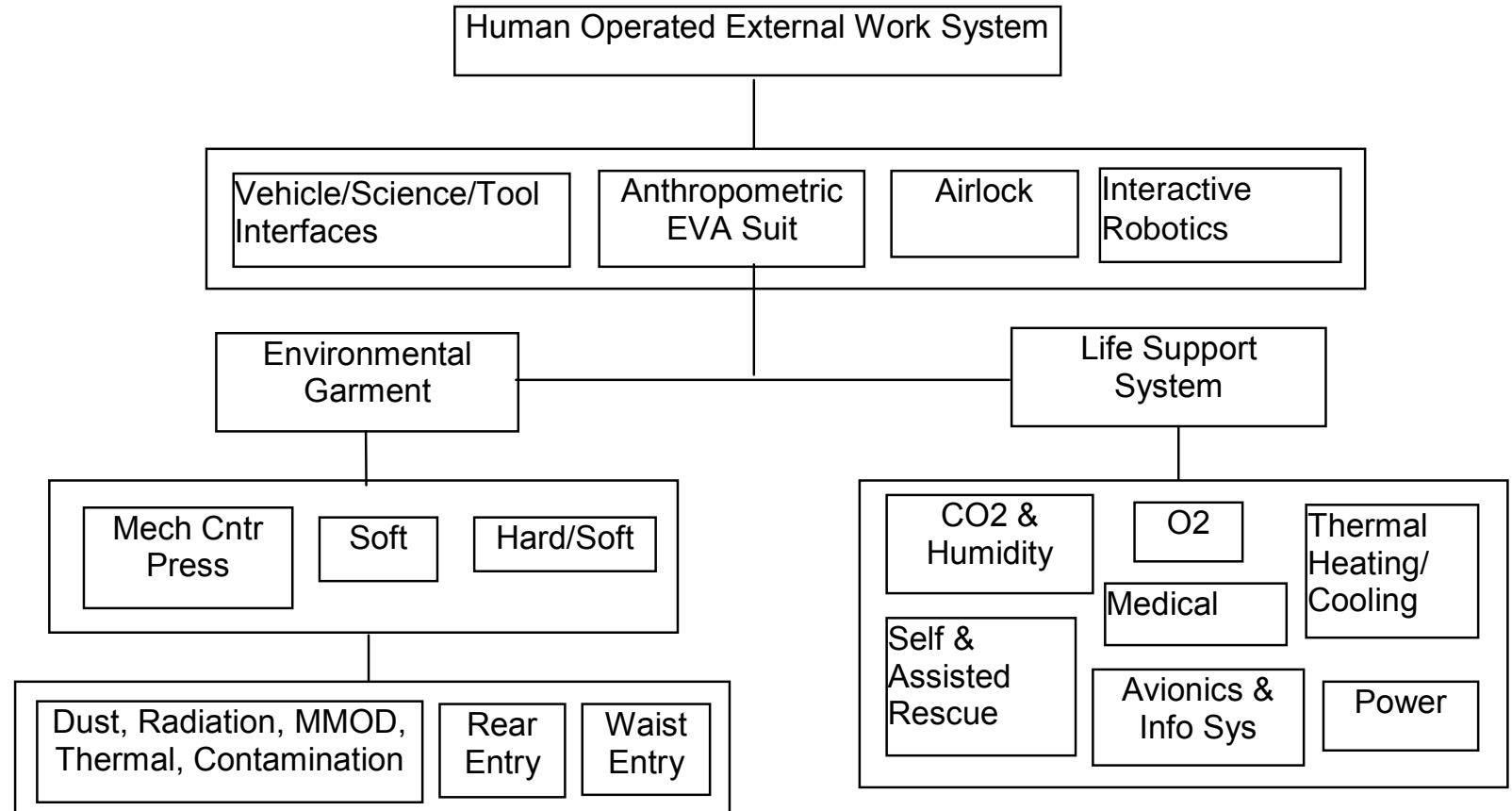

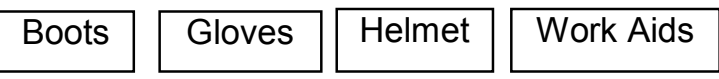



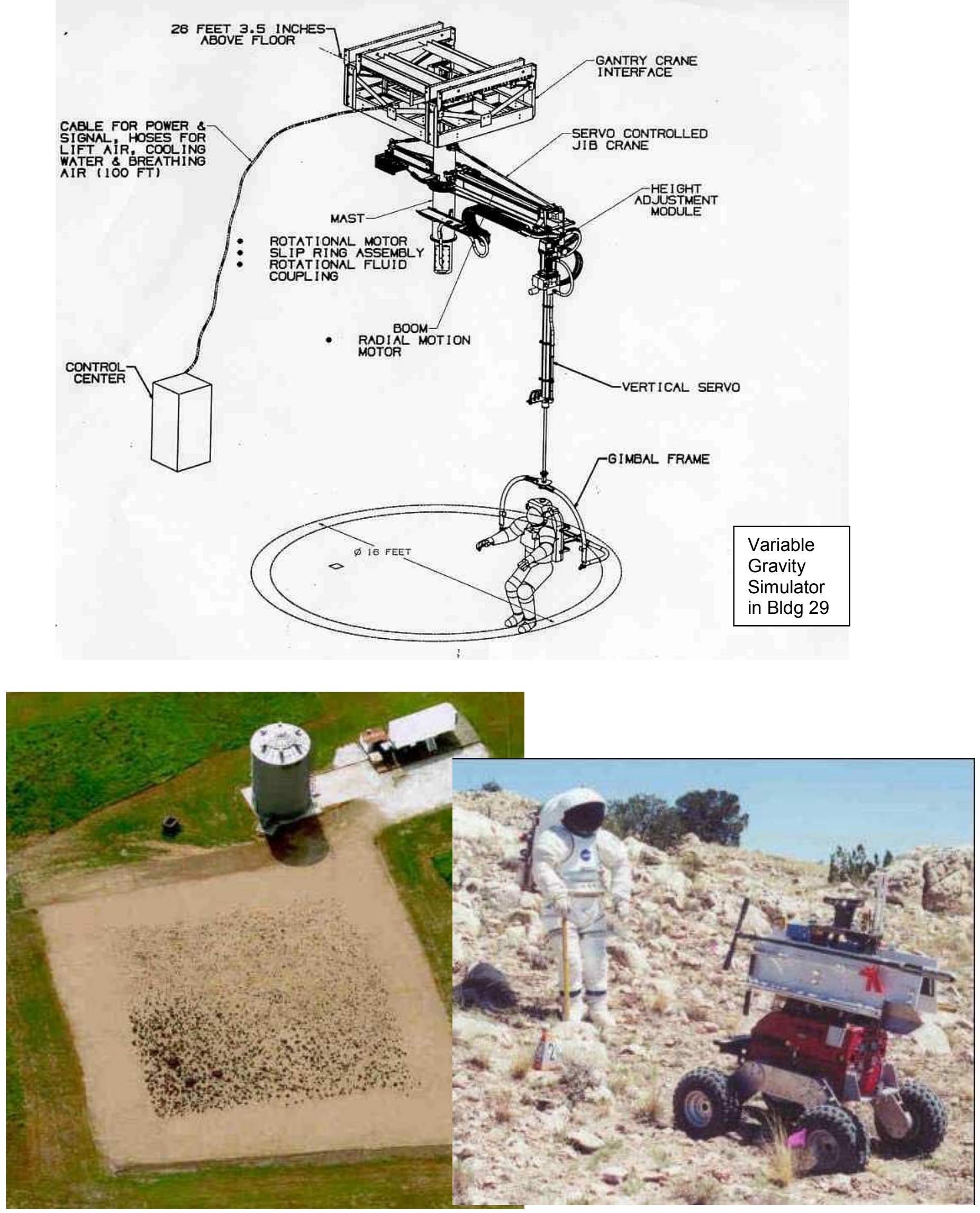